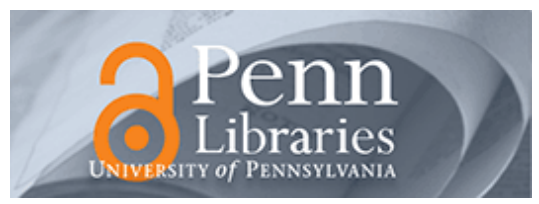

University of Pennsylvania ScholarlyCommons

December 2002

\title{
Radiation from a Traveling-Wave Current Sheet at the Interface between a Conventional Material and a Metamaterial with Negative Permittivity and Permeability
}

\author{
Andrea Alù \\ University of Pennsylvania \\ Nader Engheta \\ University of Pennsylvania, engheta@ee.upenn.edu
}

Follow this and additional works at: https://repository.upenn.edu/ese_papers

\section{Recommended Citation}

Andrea Alù and Nader Engheta, "Radiation from a Traveling-Wave Current Sheet at the Interface between a Conventional Material and a Metamaterial with Negative Permittivity and Permeability", . December 2002.

Postprint version. Published in Microwave and Optical Technology Letters, Volume 35, Issue 6, December 20, 2002, pages 460-463.

Publisher URL: http://www3.interscience.wiley.com/cgi-bin/jissue/101019882

This paper is posted at ScholarlyCommons. https://repository.upenn.edu/ese_papers/278

For more information, please contact repository@pobox.upenn.edu. 


\title{
Radiation from a Traveling-Wave Current Sheet at the Interface between a Conventional Material and a Metamaterial with Negative Permittivity and Permeability
}

\author{
Abstract \\ In this note, we present the analysis for the radiation from a traveling-wave infinitely-extent sheet of \\ monochromatic electric current that is placed at the interface between a conventional lossless dielectric \\ and a lossless material possessing negative real permittivity and permeability. The field distributions and \\ the direction of the Poynting vectors in both half spaces are discussed, and some physical remarks are \\ provided. A brief note about launching Zenneck waves by a line current along this interface is also \\ mentioned.

\section{Keywords} \\ metamaterials, negative index of refraction, negative permittivity, negative permeability, left-handed \\ medium, antenna

\section{Comments} \\ Postprint version. Published in Microwave and Optical Technology Letters, Volume 35, Issue 6, December \\ 20, 2002, pages 460-463. \\ Publisher URL: http://www3.interscience.wiley.com/cgi-bin/jissue/101019882
}




\title{
Radiation from a Traveling-Wave Current Sheet at the Interface between a Conventional Material and a Metamaterial with Negative Permittivity and Permeability
}

\author{
Andrea Alu' and Nader Engheta \\ University of Pennsylvania \\ Department of Electrical and System Engineering \\ Philadelphia, Pennsylvania 19104-6390 \\ E-mail: andreaal@ee.upenn.edu and engheta@ee.upenn.edu \\ URL: http://www.ee.upenn.edu/ engheta/
}

\begin{abstract}
In this note, we present the analysis for the radiation from a traveling-wave infinitely-extent sheet of monochromatic electric current that is placed at the interface between a conventional lossless dielectric and a lossless material possessing negative real permittivity and permeability. The field distributions and the direction of the Poynting vectors in both half spaces are discussed, and some physical remarks are provided. A brief note about launching Zenneck waves by a line current along this interface is also mentioned.
\end{abstract}

Keywords: Metamaterials, negative index of refraction, negative permittivity, negative permeability, left-handed medium, antenna.

\section{Introduction}

The topic of complex materials in which both permittivity and permeability possess negative values at some frequencies has been the subject of considerable attention recently [1-11]. The history of this idea dates back to Veselago, who in 1967 theoretically studied monochromatic plane wave propagation in a material whose permittivity and permeability he assumed to be simultaneously negative at a given frequency [6]. Recently, there has been a renewed interest in this type of material since Smith, Schultz and Shelby in their research group at UC San Diego constructed such a composite medium for the microwave regime, and experimentally showed the presence of anomalous refraction in this medium [1]. There have been several names suggested for this type of materials, such as "left-handed" materials (see e.g., [6]), materials with negative refractive index (see e.g., [3]), "double-negative (DNG)" media [8], "backward" (BW) media [7], to name a few. One of the interesting electromagnetic features of these media is the fact that for a monochromatic uniform plane wave in such a medium the direction of the Poynting vector is antiparallel with respect to the direction of phase velocity. Owing to this feature, one can envision various potential applications for DNG materials. Engheta recently introduced theoretically the idea of compact cavity 
resonators in which a combination of a slab of conventional material and a slab of DNG material is inserted [10], and in his theoretical analysis he showed that a slab of DNG metamaterial can act as a phase compensator/conjugator, and thus by combining such a slab with another slab made of a conventional dielectric material one can, in principle, have a 1-D cavity resonator whose dispersion relation may not depend on the sum of thicknesses of the interior materials filling this cavity, but instead it depends on the ratio of these thicknesses [10].

In this brief note, we analyze theoretically the electromagnetic radiation from a traveling-wave thin current sheet located at the interface between a conventional dielectric half space and a DNG material half space. This study can be informative towards any analysis and understanding of source radiation in structures involving DNG materials. In this analysis, we conceptually assume both half spaces to be lossless and, therefore, the material parameters are taken to be real-valued quantities. ${ }^{1}$

\section{Formulation of the Problem}

Consider two semi-infinite regions in a Cartesian coordinate system $(x, y, z)$; one is the half space $y>0$ which is filled with a conventional lossless medium with real permittivity and permeability $\varepsilon_{1}>0$ and $\mu_{1}>0$; and the other is the half space $y<0$ filled a DNG medium with parameters $\varepsilon_{2}<0$ and $\mu_{2}<0$. (See Fig. 1) In analogy with the terminology "DNG" introduced in [8], for the sake of brevity we call the conventional medium with positive permittivity and permeability a "double-positive" (DPS) medium.

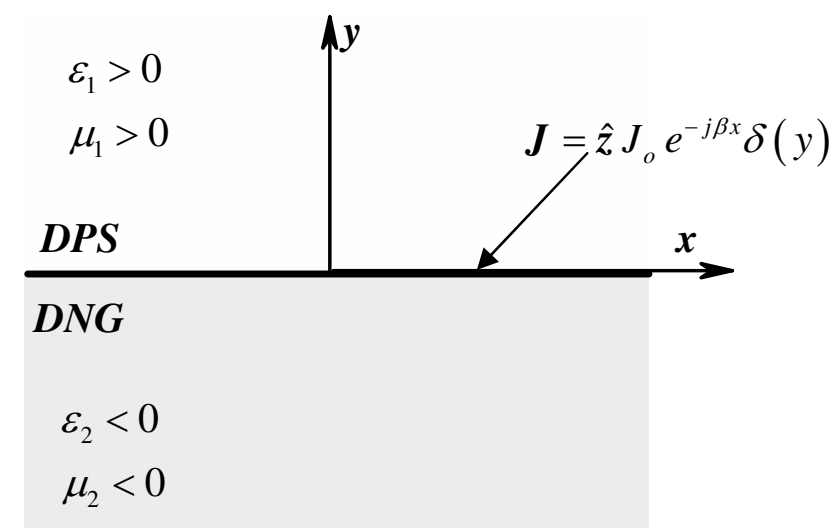

Fig. 1. Geometry of the problem. The term "DNG" stands for "double-negative" medium [8], i.e., a medium with negative permittivity and permeability, while the term "DPS", in analogy with DNG, stands for "double-positive" medium, i.e., a medium with positive permittivity and permeability. An infinitely extent thin sheet of surface current is located at the interface between the DNG and DPS media.

${ }^{1}$ We realize that strictly speaking, no material (except the vacuum in the classical sense) is dispersionless, and therefore the Kramers-Kronig relations require the inclusion of dissipation. However, in our analysis here, we assume that the dissipation is negligible at the frequency of monochromatic radiation of interest. 
An infinitely-extent thin sheet of monochromatic electric current is located at $y=0$, the interface between the DNG and DPS half spaces. Assuming the time dependence to be $e^{j \omega t}$, the density of this current sheet can be described as

$$
\boldsymbol{J}=\hat{z} J_{o} e^{-j \beta x} \delta(y)
$$

where $J_{o}$ is the strength of the surface current density, $\beta$ is the rate of the linear phase change in the $x$ direction, $\delta(\cdot)$ is the Dirac delta function, and $\hat{z}$ is the unit vector in the $z$ direction. Here we assume the direction of the current flow is along $z$, while the direction of phase variation of this traveling-wave current is along $x$. (At the end of this note, we will give the results for the case of the current sheet with the current flow and the direction of phase variation both being along the $x$ direction.)

The electromagnetic fields radiated from the current distribution given in Eq. (1) possess the electric field $\boldsymbol{E}=\hat{z} E_{z}$ where the scalar quantity $E_{z}$ satisfies the following equations in the two semi-infinite regions

$$
\begin{array}{ll}
\left(\nabla^{2}+\omega^{2} \mu_{1} \varepsilon_{1}\right) E_{z}=0 & \text { for } y>0 \\
\left(\nabla^{2}+\omega^{2} \mu_{2} \varepsilon_{2}\right) E_{z}=0 & \text { for } y<0
\end{array}
$$

with the current sheet located at $y=0$. This case we refer to as the transverse electric (TE) case. Due to our assumption stated earlier, we have $\varepsilon_{1} \mu_{1}>0$ and $\varepsilon_{2} \mu_{2}>0$, and thus the wavenumbers $\omega \sqrt{\mu_{1} \varepsilon_{1}}$ and $\omega \sqrt{\mu_{2} \varepsilon_{2}}$ are real quantities. From Eqs. (2) and (3), the expressions for $E_{z}$ can be written as

$$
E_{z}= \begin{cases}A_{1} e^{-j \beta x} e^{-j \sqrt{\omega^{2} \varepsilon_{1} \mu_{1}-\beta^{2}} y}+B_{1} e^{-j \beta x} e^{+j \sqrt{\omega^{2} \varepsilon_{1} \mu_{1}-\beta^{2}} y} & y>0 \\ A_{2} e^{-j \beta x} e^{-j \sqrt{\omega^{2} \varepsilon_{2} \mu_{2}-\beta^{2}} y}+B_{2} e^{-j \beta x} e^{+j \sqrt{\omega^{2} \varepsilon_{2} \mu_{2}-\beta^{2}} y} & y<0\end{cases}
$$

The magnetic fields can be derived from the Maxwell curl equation $\nabla \times \boldsymbol{E}=-j \omega \mu \boldsymbol{H}$. The four unknown coefficients can, in principle, be obtained by applying the boundary conditions at $y=0$

$$
\begin{gathered}
E_{z}(y=+0)=E_{z}(y=-0) \\
\hat{\boldsymbol{y}} \times[\boldsymbol{H}(y=+0)-\boldsymbol{H}(y=-0)]=\hat{z} J_{o} e^{-j \beta x}
\end{gathered}
$$

and the radiation conditions at $y= \pm \infty$. However, care must be taken when we analyze the wave in the DNG half space, since in the DNG medium the direction of the Poynting vector is antiparallel with respect to the direction of the phase flow. This point is described below. 
In the DPS half space $(y>0)$, the phase flow vector and the Poynting vector are parallel, and thus both the phase velocity vector and the Poynting vector are propagating away from the source, when $\beta<\omega \sqrt{\varepsilon_{1} \mu_{1}}$. Therefore, the coefficient $B_{1}$ should be taken to be identically zero, and we are left with the term $A_{1} e^{-j \beta x} e^{-j \sqrt{\omega^{2} \varepsilon_{1} \mu_{1}-\beta^{2}} y}$. When $\beta>\omega \sqrt{\varepsilon_{1} \mu_{1}}$, the proper sign of square root in the expression $\sqrt{\omega^{2} \varepsilon_{1} \mu_{1}-\beta^{2}}$ should be chosen in order to ensure zero fields at $y=+\infty$. Therefore, for $\beta>\omega \sqrt{\varepsilon_{1} \mu_{1}}$ we should have $\sqrt{\omega^{2} \varepsilon_{1} \mu_{1}-\beta^{2}}=-j \sqrt{\beta^{2}-\omega^{2} \varepsilon_{1} \mu_{1}}$, and we then have the term $A_{1} e^{-j \beta x} e^{-\sqrt{\beta^{2}-\omega^{2} \varepsilon_{1} \mu_{1} y}}$. In this case, the fields decay exponentially away from the source, as expected. In either case, no power will come back towards the source from infinity, since there is no reflected wave.

In the DNG half-space $(y<0)$, however, as was mentioned earlier, the phase flow vector and the Poynting vector are antiparallel. As a result, when $\beta<\omega \sqrt{\varepsilon_{2} \mu_{2}}$ while the Poynting vector should be pointed away from the source, the phase velocity vector should be pointed towards the source, as depicted in Fig. 2. Therefore, when $\beta<\omega \sqrt{\varepsilon_{2} \mu_{2}}$ the coefficient $B_{2}$ should be taken to be zero, and we are left with the term $A_{2} e^{-j \beta x} e^{-j \sqrt{\omega^{2} \varepsilon_{2} \mu_{2}-\beta^{2} y}}$. It is also important to note that in the DNG half space the radiation power flows away from the source; however, unlike the case of DPS medium, here the $x$ component of the Poynting vector is pointed in the sense opposite to the direction of the phase change of the current along the $x$ axis, as seen in Fig.2. When $\beta>\omega \sqrt{\varepsilon_{2} \mu_{2}}$ we should have $\sqrt{\omega^{2} \varepsilon_{2} \mu_{2}-\beta^{2}}=j \sqrt{\beta^{2}-\omega^{2} \varepsilon_{2} \mu_{2}}$ in order to guarantee zero field at $y=-\infty$, and we then have the term $A_{2} e^{-j \beta x} e^{\sqrt{\beta^{2}-\omega^{2} \varepsilon_{2} \mu_{2} y}}$.

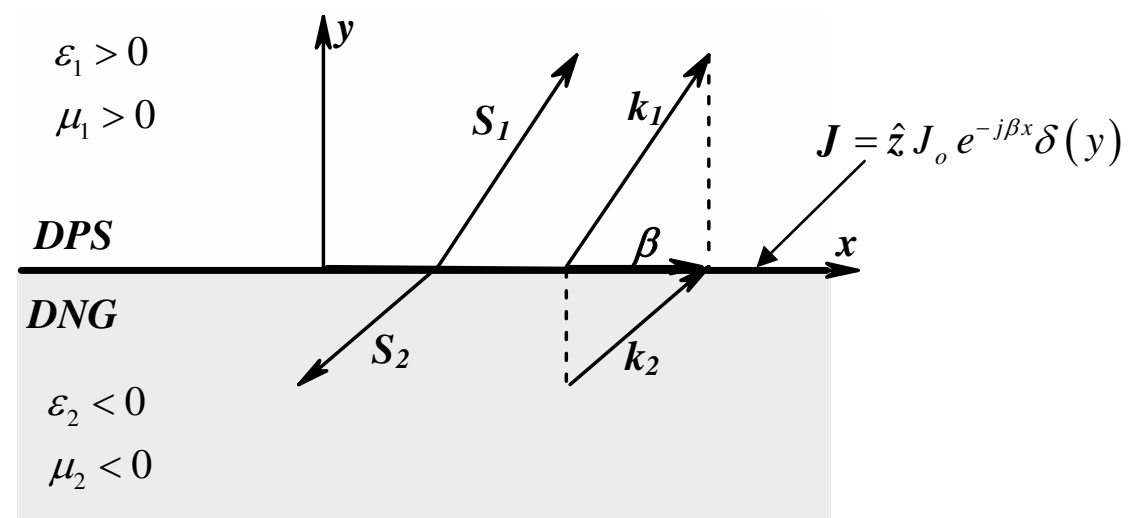

Fig. 2. Schematic representation of the directions of wave vectors $k_{1}$ and $k_{2}$, and the Poynting vectors $S_{1}$ and $S_{2}$. In the DPS medium, vectors $k_{1}$ and $S_{1}$ are parallel, while in the DNG medium the vectors $k_{2}$ and $S_{2}$ are antiparallel. The directions of power flow of the electromagnetic radiation from the monochromatic traveling-wave current sheet $\boldsymbol{J}$ shown in Eq. (1) are shown as the vectors $S_{1}$ and $S_{2}$. 
Taking into account the above issues and applying the boundary conditions, we obtain the following expressions for the electric and magnetic fields and the complex Poynting vectors in the two regions:

$$
\begin{aligned}
& \boldsymbol{E}=\hat{\boldsymbol{z}}\left\{\begin{array}{cc}
-\frac{J_{o} \omega e^{-j \beta x} e^{-j k_{t 1} y}}{\frac{k_{t 1}}{\mu_{1}}-\frac{k_{t 2}}{\mu_{2}}} & y>0 \\
-\frac{J_{o} \omega e^{-j \beta x} e^{-j k_{t 2} y}}{k_{t 1}-\frac{k_{t 2}}{\mu_{1}}} & y<0
\end{array}\right. \\
& \boldsymbol{H}=\hat{\boldsymbol{x}}\left\{\begin{array}{c}
-\frac{J_{o} e^{-j \beta x} e^{-j k_{t 1} y}}{1-\frac{\mu_{1} k_{t 2}}{\mu_{2} k_{t 1}}} \\
\frac{J_{o} e^{-j \beta x} e^{-j k_{t 2} y}}{1-\frac{\mu_{2} k_{t 1}}{\mu_{1} k_{t 2}}}
\end{array}+\hat{\boldsymbol{y}}\left\{\begin{array}{cc}
\frac{J_{o} \beta e^{-j \beta x} e^{-j k_{t 1} y}}{k_{t 1}-\frac{\mu_{1}}{\mu_{2}} k_{t 2}} & y>0 \\
-\frac{J_{o} \beta e^{-j \beta x} e^{-j k_{t 2} y}}{k_{t 2}-\frac{\mu_{2}}{\mu_{1}} k_{t 1}} & y<0
\end{array}\right.\right.
\end{aligned}
$$

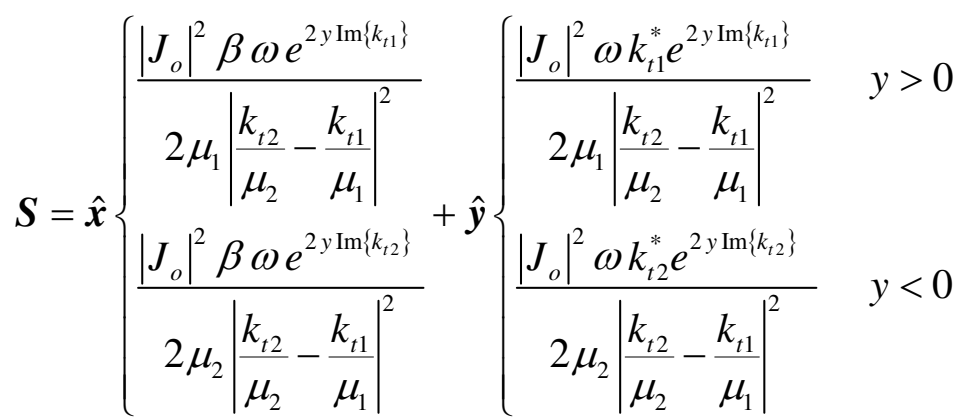

where $k_{t 1}$ and $k_{t 2}$ are shorthand for $\sqrt{\omega^{2} \varepsilon_{1} \mu_{1}-\beta^{2}}$ and $\sqrt{\omega^{2} \varepsilon_{2} \mu_{2}-\beta^{2}}$ when $\beta<\omega \sqrt{\varepsilon_{1} \mu_{1}}$ and $\beta<\omega \sqrt{\varepsilon_{2} \mu_{2}}$, respectively. Note that in the case of $\beta>\omega \sqrt{\varepsilon_{1} \mu_{1}}$ and/or $\beta>\omega \sqrt{\varepsilon_{2} \mu_{2}}$ the quantities $k_{t 1}$ and $k_{t 2}$ should be written as $k_{t 1}=-j \sqrt{\beta^{2}-\omega^{2} \varepsilon_{1} \mu_{1}}$ and $k_{t 2}=j \sqrt{\beta^{2}-\omega^{2} \varepsilon_{2} \mu_{2}}$, as was discussed earlier.

It is worth emphasizing that when $\beta>\omega \sqrt{\varepsilon_{2} \mu_{2}}$ our choice of sign for the term $k_{t 2}$ in the DNG half space leads to a physically meaningful decaying exponential as $y \rightarrow-\infty$, which is square-integrable. However, if one had chosen the opposite sign, as was done in Ref. [4], one would have obtained here a "growing" exponential as $y \rightarrow-\infty$, which would have possessed an $x$-directed real-valued Poynting vector that would have grown as $y \rightarrow-\infty$. This would have clearly been a non-physical outcome, since in such 
a situation the $x$-directed time-averaged power flux density would have been larger as one would have moved farther away from the source at $y=0$, and this is not physically possible.

It is important to note that in the case of propagating waves (i.e., when $\beta<\omega \sqrt{\varepsilon_{1} \mu_{1}}$ and $\beta<\omega \sqrt{\varepsilon_{2} \mu_{2}}$ ), since $\mu_{2}<0$ the denominators in the field expressions, Eqs. (6) and (7), cannot become zero. However, when $\beta>\omega \sqrt{\varepsilon_{1} \mu_{1}}$ and $\beta>\omega \sqrt{\varepsilon_{2} \mu_{2}}$, due to the choice of the square root mentioned earlier, the field quantities will become infinitely large if the following condition is satisfied

$$
\mu_{1} \sqrt{\beta^{2}-\omega^{2} \varepsilon_{2} \mu_{2}}+\mu_{2} \sqrt{\beta^{2}-\omega^{2} \varepsilon_{1} \mu_{1}}=0
$$

This conceptual singularity can be explained and justified in the following argument.

In the case where $\beta<\min \left\{\omega \sqrt{\varepsilon_{1} \mu_{1}}, \omega \sqrt{\varepsilon_{2} \mu_{2}}\right\}$, both components of the Poynting vector given in Eq. (8) in each region are real-valued quantities; the $y$-components are pointed away from the source and the $x$-components in two regions are antiparallel. In fact, the power flux density per unit area flowing out of a closed surface containing any segment of the current source can be evaluated as

$$
P_{r a d}=\left.\boldsymbol{S} \cdot \hat{\boldsymbol{y}}\right|_{y>0}-\left.\boldsymbol{S} \cdot \hat{\boldsymbol{y}}\right|_{y<0}=\frac{\left|J_{o}\right|^{2} \omega}{2\left(\frac{k_{t 1}}{\mu_{1}}-\frac{k_{t 2}}{\mu_{2}}\right)}
$$

which, as expected, equals the power density emitted from the source, $P_{s}$

$$
P_{s}=-\frac{1}{2} \boldsymbol{E} \cdot \boldsymbol{J}^{*}=\frac{\left|J_{o}\right|^{2} \omega}{2\left(\frac{k_{t 1}}{\mu_{1}}-\frac{k_{t 2}}{\mu_{2}}\right)}=P_{\mathrm{rad}}
$$

In the case where $\beta>\max \left\{\omega \sqrt{\varepsilon_{1} \mu_{1}}, \omega \sqrt{\varepsilon_{2} \mu_{2}}\right\}$, both $k_{t 1}$ and $k_{t 2}$ are imaginary, and thus the $y$-components of the Poynting vectors are imaginary and carry no time-averaged power away from the source. The $x$-components of the Poynting vectors in both regions are, however, real-valued quantities, and they represent power of the waves moving along the boundary and "hugging" the current source. These waves possess $x$-directed Poynting vectors that are antiparallel. From the knowledge of Eq. (8), the total timeaveraged power flow along the $x$ axis, across any plane parallel with the $y$-z plane in each of the two half spaces, can be given as 


$$
\begin{aligned}
& P_{1}=\int_{0}^{\infty} \boldsymbol{R e}(S) \cdot \hat{\boldsymbol{x}} d y=\frac{\left|J_{o}\right|^{2} \beta \omega}{4 \mu_{1}\left|k_{t 1}\right|\left|\frac{k_{t 2}}{\mu_{2}}-\frac{k_{t 1}}{\mu_{1}}\right|^{2}} \\
& P_{2}=\int_{-\infty}^{0} \operatorname{Re}(S) \cdot \hat{\boldsymbol{x}} d y=\frac{\left|J_{o}\right|^{2} \beta \omega}{4 \mu_{2}\left|k_{t 2}\right| \frac{k_{t 2}}{\mu_{2}}-\left.\frac{k_{t 1}}{\mu_{1}}\right|^{2}}
\end{aligned}
$$

and the net total time-averaged power then becomes

$$
P=P_{1}+P_{2}=\frac{\left|J_{o}\right|^{2} \beta \omega \mu_{1} \mu_{2}}{4\left|\mu_{1} k_{t 2}-\mu_{2} k_{t 1}\right|^{2}}\left(\frac{\mu_{1}}{\left|k_{t 2}\right|}+\frac{\mu_{2}}{\left|k_{t 1}\right|}\right)
$$

with $k_{t 1}=-j \sqrt{\beta^{2}-\omega^{2} \varepsilon_{1} \mu_{1}}$ and $k_{t 2}=j \sqrt{\beta^{2}-\omega^{2} \varepsilon_{2} \mu_{2}}$. This net power may vanish if $\mu_{1}\left|k_{t 1}\right|=-\mu_{2}\left|k_{t 2}\right|$, which is different from the condition given in Eq. (9). It is important to note that Eq. (9) is indeed the condition for existence of source-free Zenneck waves at the boundary between a DNG and a DPS medium, as can be deduced from the work of Lindell et al. [7]. Therefore, if one wants to launch a Zenneck wave along this interface, one can put a thin line current along the $z$ axis at the interface, i.e., $\boldsymbol{J}_{\text {line }}=\hat{z} I_{o} \delta(x) \delta(y)$. Such a line current can be expanded using the following Fourier transform:

$$
\boldsymbol{J}_{\text {line }}=\hat{z} I_{o} \delta(x) \delta(y)=\hat{z} \frac{I_{o}}{2 \pi} \int_{-\infty}^{\infty} d \beta e^{-j \beta x} \delta(y) .
$$

The fields due to this line current can then be given as

$$
\begin{aligned}
& \boldsymbol{E}_{\text {line }}=\frac{I_{o}}{2 \pi J_{o}} \int_{-\infty}^{+\infty}\{\text { Eq. (6) }\} d \beta \\
& \boldsymbol{H}_{\text {line }}=\frac{I_{o}}{2 \pi J_{o}} \int_{-\infty}^{+\infty}\{\text { Eq. (7) }\} d \beta
\end{aligned}
$$

where Eq. (6) and Eq. (7) are field quantities due to the current sheet of $\boldsymbol{J}=\hat{z} J_{o} e^{-j \beta x} \delta(y)$. In Eq. (15), the radiation fields are due to the integration over the interval $|\beta|<\max \left\{\omega \sqrt{\varepsilon_{1} \mu_{1}}, \omega \sqrt{\varepsilon_{2} \mu_{2}}\right\}$ and can be calculated from the knowledge of Eqs. (6) and (7). In addition to the radiation fields, the integrals given in Eq. (15) will also provide the surface wave due to the residue contribution, which occurs at the poles of Eqs. (6) and (7). These poles indeed satisfy Eq. (9), which leads to the following $\beta$ : 


$$
\beta_{\text {surf }}= \pm \omega \sqrt{\frac{\varepsilon_{1} / \mu_{1}-\varepsilon_{2} / \mu_{2}}{1 / \mu_{1}^{2}-1 / \mu_{2}^{2}}}
$$

If this $\beta_{\text {surf }}$ is real and if $\left|\beta_{\text {surf }}\right|>\max \left\{\omega \sqrt{\varepsilon_{1} \mu_{1}}, \omega \sqrt{\varepsilon_{2} \mu_{2}}\right\}$, the electric field of such a surface wave can then be calculated by means of the residue theorem and can be expressed as:

$$
\begin{aligned}
& \boldsymbol{E}_{\text {surf }}=\hat{\boldsymbol{z}} \omega I_{o} \frac{e^{-j \beta_{\text {surf }} x}}{\left.\frac{\partial}{\partial \beta}\left[\frac{\left|k_{t 1}\right|}{\mu_{1}}+\frac{\left|k_{t 2}\right|}{\mu_{2}}\right]\right|_{\beta=\beta_{\text {sur }}}}\left\{\begin{array}{ll}
e^{-\sqrt{\beta_{\text {surf }}^{2}-\omega^{2} \varepsilon_{1} \mu_{1} y}} & y>0 \\
e^{\sqrt{\beta_{\text {surf }}^{2}-\omega^{2} \varepsilon_{2} \mu_{2} y}} & y<0
\end{array}=\right. \\
& =\hat{z} \omega I_{o} \frac{e^{-j \beta_{\text {surf }} x} \begin{cases}e^{-\sqrt{\beta_{\text {suf }}^{2}-\omega^{2} \varepsilon_{1} \mu_{1} y}} & y>0 \\
e^{\sqrt{\beta_{\text {surf }}^{2}-\omega^{2} \varepsilon_{2} \mu_{2} y}} & y<0\end{cases} }{\beta_{\text {surf }}\left(\frac{1}{\mu_{1} \sqrt{\beta_{\text {surf }}^{2}-\omega^{2} \varepsilon_{1} \mu_{1}}}+\frac{1}{\mu_{2} \sqrt{\beta_{\text {surf }}^{2}-\omega^{2} \varepsilon_{2} \mu_{2}}}\right)} .
\end{aligned}
$$

The magnetic field of such a surface wave can be calculated similarly. So from a line current source (Eq. (14)) this Zenneck wave is launched. Now if we put an infinite number of such line current sources next to each other arranged such that they form a current sheet of Eq. (1) with $\beta=\beta_{\text {surf }}$, there will be an infinite number of Zenneck waves each being launched from each line current source, and since $\beta=\beta_{\text {surf }}$ they all would be added constructively. The amplitude of such combined Zenneck wave would therefore be infinite. This justifies and describes why the condition given in Eq. (9) leads to singularity in Eqs. (6) and (7).

It is worth noting that Eq. (9) in general may provide the condition for a single Zenneck wave to propagate with $\beta_{\text {surf }}$ (as given in Eq. (16)) along the interface between the DNG and the DPS media. However, for a particular case of $\left\{\begin{array}{l}\varepsilon_{2}=-\varepsilon_{1} \\ \mu_{2}=-\mu_{1}\end{array}\right.$, Eq. (9) is satisfied for any given $\beta$ as long as $|\beta|>\max \left\{\omega \sqrt{\varepsilon_{1} \mu_{1}}, \omega \sqrt{\varepsilon_{2} \mu_{2}}\right\}$. But in this case, such surface waves will carry zero net power, since according to Eq. (13) $P=0$ in this case for any $\beta$. In this scenario, two oppositely directed, but equal, power fluxes propagate along the $x$ axis, effectively behaving similar to a "standing wave" in a cavity or a resonant circuit. We also note that in this case, the denominator of Eq. (17) vanishes, resembling a situation in which a resonant parallel L-C circuit is excited by a monochromatic parallel current source oscillating at the resonant frequency $\omega=1 / \sqrt{L C}$, resulting in an infinitely large voltage in the L-C circuit. (Or equivalently a resonant series L-C circuit being excited by a monochromatic series voltage source at the resonant 
frequency resulting in an infinite current in the series L-C circuit.) This also somewhat resembles a situation in which an ideal lossless cavity resonator is excited by a forced current element, oscillating at the resonant frequency of the cavity, located at an interior point with a non-zero value of the electric field of the cavity mode. In such a "driven" cavity, this forced oscillation leads to infinitely large values for the electric and magnetic fields within the cavity.

Similar analysis can be performed for the case of current distribution of the form $\boldsymbol{J}=\hat{\boldsymbol{x}} J_{o} e^{-j \beta x} \delta(y)$. In this case, which is the transverse magnetic (TM) case, the corresponding electromagnetic fields and related Poynting vectors can be given as:

$$
\begin{aligned}
& \boldsymbol{E}=\hat{\boldsymbol{x}}\left\{\begin{array}{l}
\frac{J_{o} e^{-j \beta x} e^{-j k_{t 1} y}}{\omega\left(\frac{\varepsilon_{2}}{k_{t 2}}-\frac{\varepsilon_{1}}{k_{t 1}}\right)} \\
\frac{J_{o} e^{-j \beta x} e^{-j k_{t 2} y}}{\omega\left(\frac{\varepsilon_{2}}{k_{t 2}}-\frac{\varepsilon_{1}}{k_{t 1}}\right)}
\end{array}+\hat{\boldsymbol{y}}\left\{\begin{array}{cc}
\frac{J_{o} \beta e^{-j \beta x} e^{-j k_{t 1} y}}{\omega\left(\varepsilon_{1}-\varepsilon_{2} \frac{k_{t 1}}{k_{t 2}}\right)} & y>0 \\
-\frac{J_{o} \beta e^{-j \beta x} e^{-j k_{t 2} y}}{\omega\left(\varepsilon_{1} \frac{k_{t 2}}{k_{t 1}}-\varepsilon_{2}\right)} & y<0
\end{array}\right.\right. \\
& \boldsymbol{H}=\hat{\boldsymbol{z}}\left\{\begin{array}{cc}
\frac{J_{o} e^{-j \beta x} e^{-j k_{t 1} y}}{1-\frac{\varepsilon_{2} k_{t 1}}{\varepsilon_{1} k_{t 2}}} & y>0 \\
\frac{J_{o} e^{-j \beta x} e^{-j k_{t 2} y}}{\frac{\varepsilon_{1} k_{t 2}}{\varepsilon_{2} k_{t 1}}-1} & y<0
\end{array}\right. \\
& \boldsymbol{S}=\hat{\boldsymbol{x}}\left\{\begin{array}{l}
\frac{\left|J_{o}\right|^{2} e^{2 y \operatorname{Im}\left\{k_{t 1}\right\}} \beta \varepsilon_{1}}{2 \omega\left|\varepsilon_{1}-\varepsilon_{2} \frac{k_{t 1}}{k_{t 2}}\right|^{2}} \\
\frac{\left|J_{o}\right|^{2} e^{2 y \operatorname{Im}\left\{k_{t 2}\right\}} \beta \varepsilon_{2}}{2 \omega\left|\varepsilon_{2}-\varepsilon_{1} \frac{k_{t 2}}{k_{t 1}}\right|^{2}}
\end{array}+\hat{\boldsymbol{y}}\left\{\begin{array}{l}
\frac{\left|J_{o}\right|^{2} e^{2 y \operatorname{Im}\left\{k_{t 1}\right\}} k_{t 1} \varepsilon_{1}}{2 \omega\left|\varepsilon_{1}-\varepsilon_{2} \frac{k_{t 1}}{k_{t 2}}\right|^{2}} \\
\frac{\left|J_{o}\right|^{2} e^{2 y \operatorname{Im}\left\{k_{t 2}\right\}} k_{t 2} \varepsilon_{2}}{2 \omega\left|\varepsilon_{2}-\varepsilon_{1} \frac{k_{t 2}}{k_{t 1}}\right|^{2}}
\end{array}\right.\right.
\end{aligned}
$$

with $k_{t 1}=\sqrt{\omega^{2} \mu_{1} \varepsilon_{1}-\beta^{2}}$ and $k_{t 2}=\sqrt{\omega^{2} \mu_{2} \varepsilon_{2}-\beta^{2}}$ for $\beta<\omega \sqrt{\mu_{1} \varepsilon_{1}}$ and $\beta<\omega \sqrt{\mu_{2} \varepsilon_{2}}$, and $k_{t 1}=-j \sqrt{\beta^{2}-\omega^{2} \mu_{1} \varepsilon_{1}}$ and $k_{t 2}=j \sqrt{\beta^{2}-\omega^{2} \mu_{2} \varepsilon_{2}}$ for $\beta>\omega \sqrt{\mu_{1} \varepsilon_{1}}$ and $\beta>\omega \sqrt{\mu_{2} \varepsilon_{2}}$, and other quantities similar to the ones defined earlier for the transverse electric (TE) case. Similar considerations can be mentioned in this TM case. 
In summary, we have presented our analysis of the electromagnetic radiation from an infinitely extent monochromatic thin sheet of current located at the interface between a DPS medium and a DNG medium. The results of this analysis show that the Poynting vectors in the two media are pointed away from the source; however, the $x$-components of the two Poynting vectors are in opposite directions. This is due to the fact that in the DNG medium for a monochromatic uniform plane wave propagation the Poynting vector and the phase velocity vector are antiparallel. This result is consistent with what Veselago has conjectured about the direction of the Cerenkov radiation in the DNG media [6].

\section{Acknowledgements}

Andrea Alu' is supported by the scholarship "Isabella Sassi Bonadonna" from AEI (Associazione Elettrotecnica ed Elettronica Italiana). This work is supported in part by the Fields and Waves Laboratory, Department of Electrical and Systems Engineering, University of Pennsylvania.

\section{References}

[1] R. A. Shelby, D. R. Smith, S. Schultz, "Experimental verification of a negative index of refraction," Science, vol. 292, no. 5514, pp. 77-79, April 6, 2001.

[2] D. R. Smith, W. J. Padilla, D. C. Vier, S. C. Nemat-Nasser, and S. Schultz, "Composite medium with simultaneously negative permeability and permittivity," Phys. Rev. Lett., vol. 84, no. 18, pp. 4184-4187, 1 May 2000.

[3] D. R. Smith and N. Kroll, "Negative refractive index in left-handed materials," Phys. Rev. Lett., vol. 85, no. 14, pp. 2933-2936, 2 October 2000.

[4] J. B. Pendry, "Negative refraction makes a perfect lens," Phys. Rev. Lett., vol. 85, no. 18 , pp. 3966-3969, 30 October 2000.

[5] R. A. Shelby, D. R. Smith, S. C. Nemat-Nasser, and S. Schultz, "Microwave transmission through a two-dimensional, isotropic, left-handed metamaterial," Applied Physics Lett., vol. 78, no. 4, pp. 489-491, 22 January 2001.

[6] V. G. Veselago, "The electrodynamics of substances with simultaneously negative values of $\varepsilon$ and $\mu$," Soviet Physics Uspekhi, vol. 10, no. 4, pp. 509-514, 1968. [Usp. Fiz. Nauk, vol. 92, pp. 517-526, 1967.]

[7] I. V. Lindell, S. A. Tretyakov, K. I. Nikoskinen, and S. Ilvonen, "BW media Media with negative parameters, capable of supporting backward waves," Microwave and Optical Technology Letters, Vol. 31, No. 2, pp. 129-133, October 2001. 
[8] R. W. Ziolkowski and E. Heyman, "Wave propagation in media having negative permittivity and permeability," Phys. Rev. E., vol. 64, no. 5, 056625, October 2001.

[9] M. W. McCall, A. Lakhtakia, and W. S. Weiglhofer, "The negative index of refraction demystified", European Journal of Physics, Vol. 23, pp. 353-359, 2002.

[10] N. Engheta, "An idea for thin subwavelength cavity resonators using metamaterials with negative permittivity and permeability," IEEE Antennas and Wireless Propagation Letters, Vol. 1, No. 1, 2002, to appear on line.

[11] N. Garcia and M. Nieto-Vesperinas, "Left-Handed Materials Do Not Make a Perfect Lens," Physical Review Letters, Vol. 88, No. 20, 207403, May 20, 2002. 